\title{
EFFECT OF DIFFERENT SOURCES AND LEVELS OF SOME DIETARY BIOLOGICAL ADDITIVES ON: I-GROWTH PERFORMANCE AND PRODUCTION ECONOMY OF NILE TILAPIA FISH \\ Abdelhamid' ${ }^{1}$, A.M.; M.E. A.Seden ${ }^{2}$ and O.A.Zenhom ${ }^{2}$ \\ 1 Anim. Prod. Dept., Fac. of Agric., Al-Mansourah Univ., Al-Mansourah, Egypt. \\ ${ }^{2}$ Cent. Lab. for Aqua. Res., Abbasa, Abo-Hammad, Egypt.
}

\begin{abstract}
The present study aimed to investigate the effect of dietary graded levels of Aqua Superzyme, Garlen Allicin, and Diamond V (Original XPC) on the growth performance and economic production of Nile tilapia fish (10 $\mathrm{g}$ initial bodyweight) for 16 weeks in indoor feeding experiment. The obtained results revealed that the best treatment was the probiotic Diamond V- Original XPC at $0.5 \%$ of the diet which was responsible for the significantly $(P \leq 0.05)$ best final bodyweight, total bodyweight gain, relative growth rate, specific growth rate, survival rate, condition factor (based on total length), return and economic efficiency among all tested materials and levels. So. It is recommended to use the feed additive Diamond V-Original XPC at $0.5 \%$ of the Nile tilapia diet for improving the growth performance, survival, and production economy.

Keywords: Prebiotic - probiotic - performance - economy - tilapia
\end{abstract}

\section{INTRODUCTION}

Probiotics (specific microbial feeds with potential benefits to the host), and prebiotics (dietary components such as complex carbohydrates able to change the colonic microenvironment fostering colonization with nonenteropathogens) are areas of current interest because they offer alternatives for the management of the growing problem of multiple antibiotic resistance and overwhelming infections in the hospitalized patient (Castillo, 2008). With increasing demand for environment friendly aquaculture, the use of probiotics in aquaculture is now widely accepted (Wang et al., 2008). Hence, many studies were conducted to examine the effect of probiotics on Nile tilapia $O$. niloticus growth performance (Allam, 2007 and Marzouk et al., 2008). Also, many studies revealed the positive effects of probiotics on different $O$. niloticus stages such fry (Abdelhamid et al., 2012 and Abdel-Tawwab, 2012) and fingerlings (Mehrim, 2009 and Khalafalla, 2010). Results showed that dietary supplementation of $B$. subtilis at a dose of $1.35 \times 10^{7} \mathrm{cfu}^{-1}$ improved growth of juvenile large yellow croaker, L. crocea (Ai et al., 2011). Many products are commercially found in the local market; therefore, the present investigation aimed to compare among three different pre-and probiotics at three graded levels (besides control free of such additives) concerning their effects on growth performance and production economy of Nile tilapia fish in indoor feeding experiment for 16 weeks. 


\section{MATERIALS AND METHODS}

\section{Experimental facilities:}

Thirty glass aquaria with dimensions of $60 \times 75 \times 50 \mathrm{~cm}(\mathrm{~W}, \mathrm{~L}, \mathrm{D})$ were used at triplicates/treatment. All male mono-six (treated with $17 \alpha$ methyl-testosterone) Nile tilapia, Oreochromis niloticus, fingerlings were obtained from fish hatchery of the Cent. Lab. of Aqua. Res., Abbasa, AbouHammad. After arrival, fish were kept under the same environmental conditions and placed in fiberglass tanks for 2 weeks as adaptation period to alleviate stresses during transportation and to be adapted to new conditions. Fish were fed the control diet containing $30 \%$ crude protein for two weeks, during this period healthy fish of the same weight replaced the dead ones (Tables 1 and 2).

Fifteen fish/aquarium of the same initial body weight $(10 \mathrm{~g} / \mathrm{fish})$ were selected and randomly distributed into ten experimental treatments (3 perand/or probiotics $\times 3$ levels of each + control) in triplicates. Each aquarium was supplied with compressed air via air-stones using aquarium air pumps. Settled fish wastes with one half of aquarium's water were removed daily by siphoning and water volumes were replaced by dechlarinated aerated tap water from the storage tank. Water temperature range was $\left(26-28{ }^{\circ} \mathrm{C}\right)$. Each diet was given to fish at a rate of $3 \%$ of live body weight twice daily at 9 am and $1 \mathrm{pm}$. The appointed quantity of diet was offered to fish 6 days a week throughout the experimental period.

Fish in each aquarium were weighed biweekly and the amounts of the required feed were readjusted according to the actual body weight. The dead fish was daily recorded and removed. At the end of study, fish were individually weighed and weights and lengths were measured.

Table 1: Scheme of the experimental conditions.

\begin{tabular}{|l|c|}
\hline Treatment No. & $\mathbf{3 X 3}$ \\
\hline Replication No. & 3 \\
\hline Stocking rate, fish/aquarium & $15 \mathrm{fish}$ \\
\hline Initial weight, $\mathrm{g}$ & $10 \mathrm{~g}$ \\
\hline Aquarium dimensions, $\mathrm{cm}$ & $60 \times 75 \times 50$ \\
\hline Water value, L/aquarium & 180 \\
\hline Adaptation period, $\mathrm{d}$ & 14 \\
\hline Experimental period, $\mathrm{d}$ & 112 day \\
\hline Daily feeding rate, \% biomass & 3 \\
\hline Photoperiod, $\mathrm{h}$ & $12 / 12$ \\
\hline
\end{tabular}


Table 2: Dietary levels of the experimented pre-and/or probiotics.

\begin{tabular}{|l|c|c|c|}
\hline Treat. & $\begin{array}{c}\text { Aqua } \\
\text { Superzyme }\end{array}$ & $\begin{array}{c}\text { Garlen } \\
\text { Allicin }\end{array}$ & $\begin{array}{c}\text { Diamond V } \\
\text { (Original XPC) }\end{array}$ \\
\hline T0 & \multicolumn{3}{|c|}{ control } \\
\hline T0 & \multicolumn{3}{|c|}{ control } \\
\hline T0 & control \\
\hline T1 & $0.01 \%$ & $0.01 \%$ & $0.4 \%$ \\
\hline T1 & $0.01 \%$ & $0.01 \%$ & $0.4 \%$ \\
\hline T1 & $0.01 \%$ & $0.01 \%$ & $0.4 \%$ \\
\hline T2 & $0.02 \%$ & $0.02 \%$ & $0.5 \%$ \\
\hline T2 & $0.02 \%$ & $0.02 \%$ & $0.5 \%$ \\
\hline T3 & $0.02 \%$ & $0.02 \%$ & $0.5 \%$ \\
\hline T3 & $0.03 \%$ & $0.03 \%$ & $0.6 \%$ \\
\hline T3 & $0.03 \%$ & $0.03 \%$ & $0.6 \%$ \\
\hline
\end{tabular}

\section{Feed additives:}

Three preparations of commercial prebiotics/probiotics were used, mainly Aqua Superzyme, Garlen Allicin, and Diamond V (Original XPC) to test the effect of their graded levels on the productive performance of Nile tilapia fingerlings. The composition of these preparations, as claimed by the manufacturers, are shown in Tables 3, 4, and 5.

Table3:Minimum guaranteed analysis of Preparation No.1 (A) (Aqua Superzyme), each $1000 \mathrm{~g}$ prebiotic contain:

\begin{tabular}{|l|c|}
\hline Sodium butyrate & $\mathbf{2 5 0} \mathbf{~ g}$ \\
\hline Zinc methionise & $250 \mathrm{~g}$ \\
\hline Lipase & $200 \mathrm{~g}$ \\
\hline Phytase & $500 \mathrm{FTU}$ \\
\hline Cellulase & $200 \mathrm{U}$ \\
\hline Hylanase & $10.000 \mathrm{U}$ \\
\hline B- gluconase & $2000 \mathrm{U}$ \\
\hline Protease & $1000 \mathrm{U}$ \\
\hline Amylase & $200 \mathrm{U}$ \\
\hline Calcium carbonate & up to $1000 \mathrm{~g}$ \\
\hline
\end{tabular}

Origin: Norel- Spain \& www.norelnature.com. Importer: Mediavet CO., for import \& export 5 Al Kholfaa El Rashdeen St.,- Kafr El Sheikh- Egypt. www.mediaavet.com 
Table 4: Analysis of preparation No. 2 (Garlen Allicin), probiotic (G).

\begin{tabular}{|c|c|c|c|}
\hline Item & Specification & Result & Test Method \\
\hline \multicolumn{4}{|l|}{ Active Ingredients } \\
\hline Assay(ppm) & $\begin{array}{c}\text { Allicin content } \\
(24 \%-26 \%)\end{array}$ & $25.10 \%$ & HPLC \\
\hline \multicolumn{4}{|c|}{ Physical Control } \\
\hline Appearance & Powder & Complies & Visual \\
\hline Color & White & Complies & Visual \\
\hline Sieve Analysis & $100 \%$ thru 80 mesh & Complies & Mesh Screen \\
\hline \multicolumn{4}{|c|}{ Chemical Control } \\
\hline Heavy metals & NMT 20 ppm & Conforms & $\begin{array}{c}\text { Atomic } \\
\text { Absorption }\end{array}$ \\
\hline Arsenic(As) & NMT20 ppm & Conforms & $\begin{array}{c}\text { Atomic } \\
\text { Absorption }\end{array}$ \\
\hline Lead (pb) & NMT0.5ppm & Conforms & $\begin{array}{c}\text { Atomic } \\
\text { Absorption }\end{array}$ \\
\hline \multicolumn{4}{|c|}{ Microbiological Control } \\
\hline Total Plat Count & $10,000 \mathrm{cfn} / \mathrm{Max}$ & Conforms & AOAC \\
\hline Yeast \& Mold & $300 \mathrm{cfu} / \mathrm{g} \mathrm{Max}$ & Conforms & AOAC \\
\hline E.Coli & Negative & Negative & AOAC \\
\hline Salmonella & Negative & Negative & AOAC \\
\hline Staph Aureus & Negative & Negative & AOAC \\
\hline $\begin{array}{l}\text { Pseudomonas } \\
\text { aeruginosa }\end{array}$ & Negative & Negative & USP \\
\hline
\end{tabular}

Product Name: Allicin Powder extra(Allicin 25\%) bled of volatile oils $10 \%$. Origin: Hefe Royal Eagle- China. Importer: Mediavet CO., for import \& export 5 Al Kholfaa El Rashdeen St.,- Kafr El Sheikh- Egypt. www.mediaavet.com

\section{Experimental diets:}

Chemical composition of feedstuff used in this study is presented in Table 6. Ingredient composition of the experimental diets for the $1^{\text {st }}, 2^{\text {nd }}$ and $3^{\text {rd }}$ experiments are presented in Tables 7,8 , and 9 respectively. Fishmeal and soybean meal were used as sources of animal and plant protein sources, respectively, wheat bran and wheat milling by product were added in a constant percent for both $(15 \%$ of diet) in all the experimental diets as energy sources. Wheat milling by products served as a bender agent. Fish meals were replaced by corn starch to give a different protein levels. Vitamin and mineral premixes were added with $2 \%$ for each diet. Composition of the vitamins and minerals premix is presented in Table 10. 
Table 5:Analysis of preparation No. 3 (Diamond V (Original XPC), probiotic.

\begin{tabular}{|l|c|}
\hline Crude protein & min. $\mathbf{1 2 . 0} \%$ \\
\hline Lysine & $\min .0 .35 \%$ \\
\hline Methionine & $\min .0 .08 \%$ \\
\hline Crude fat & $\min .3 .0 \%$ \\
\hline Crude fiber & $\operatorname{max.} 6.5 \%$ \\
\hline Ash & $\operatorname{max.} 6.5 \%$ \\
\hline NFE & $\operatorname{max.~} 54.5 \%$ \\
\hline Manganese & $\min .23 \mathrm{mg} / \mathrm{Kg}$ \\
\hline Phosphorus & $\min .6000 \mathrm{mg} / \mathrm{Kg}$ \\
\hline Potassium & $\min .1 .25 \%$ \\
\hline Biotin & $\min .0 .2 \mathrm{mg} / \mathrm{Kg}$ \\
\hline Niacin & $\min .70 \mathrm{mg} / \mathrm{Kg}$ \\
\hline
\end{tabular}

Diamond V XP Yeast Culture, Diamond V Mills, Cedar Rapids, lowa, USA). Manufactured by Diamond V( Original XP) Mills, Inc. Cedar Rapids, lowa U.S.A. 52407. Importer: EGYVET-CARE \& EI-Mehala Alkobra 10 Ammar Ibn Yasser St. www. Egyvet_care@yahoo.com. Active viable microorganisms: Saccharomyces cerevisiae yeast and the media on which it was grown, consisting of ground yellow maize, homing feed, wheat middling, rye middling, diastatic malt and maize syrup, and cane molasses.

Table 6: Chemical composition (\% dry matter basis) of feedstuff used in the experimental diets.

\begin{tabular}{|c|c|c|c|c|c|c|}
\hline Ingredient & CP & EE & CF & NFE & Ash & $\begin{array}{c}\mathrm{GE}^{1} \\
\mathrm{kcal} / 100 \mathrm{~g}\end{array}$ \\
\hline Fishmeal $^{2}$ & 71.90 & 9.14 & 0.6 & 2.01 & 16.35 & 501 \\
\hline Soybean meal 3 & 48.50 & 0.90 & 3.40 & 40.70 & 6.50 & 453 \\
\hline Wheat bran 4 & 16.40 & 4.0 & 9.90 & 64.40 & 5.30 & 401 \\
\hline $\begin{array}{l}\text { Wheat milling By- } \\
\text { product } 5\end{array}$ & 17.0 & 4.30 & 8.0 & 66.10 & 4.60 & 414 \\
\hline Corn starch 6 & 0.20 & Trace & 0.08 & 99.64 & 0.080 & 420 \\
\hline
\end{tabular}

1- Calculated using factors of $5.65,9.45$ and $4.2 \mathrm{Kcal}$ per $\mathrm{g}$ of protein, fat and carbohydrates, respectively (NRC, 1993). 2- Hearing fish meal , Revisen , Co., Denmark. 3- Kafr EL- Zayyat Extracted Oils Company, Kafr EL-Zayyat, Egypt (48\%). 4-5- East Delta , Millis Co., EL-Arish, North Sinai, Egypt. 6- Egyptian company for glucose \& Corn starch industry (Qrnish El-Nile-Torah-Costatica. 
Table 7: Ingredients composition $(\mathrm{g} / 100 \mathrm{~g})$ and chemical analysis (\% dry matter basis) of the experimental diets used in the $1^{\text {st }}$ additive (A).

\begin{tabular}{|c|c|c|c|c|}
\hline \multirow[t]{2}{*}{ Ingredients } & \multicolumn{4}{|c|}{ Aqua Superzyme (A) levels } \\
\hline & $\mathbf{0}$ & A1 & A2 & A3 \\
\hline Fish meal & 10 & 10 & 10 & 10 \\
\hline Soybean meal & 44 & 44 & 44 & 44 \\
\hline Wheat milling by product & 15 & 15 & 15 & 15 \\
\hline Yellow corn & 21.38 & 21.38 & 21.38 & 21.38 \\
\hline Corn starch & 2.0 & 1.99 & 1.98 & 1.97 \\
\hline O il mixture ${ }^{1}$ & 4.62 & 4.62 & 4.62 & 4.62 \\
\hline Vitamin premix ${ }^{2}$ & 1.0 & 1.0 & 1.0 & 1.0 \\
\hline Mineral premix ${ }^{3}$ & 2.0 & 2.0 & 2.0 & 2.0 \\
\hline Aqua Superzyme & 0.0 & 0.01 & 0.02 & 0.03 \\
\hline Total & 100 & 100 & 100 & 100 \\
\hline \multicolumn{5}{|c|}{ Chemical analysis } \\
\hline Dry matter & 92.55 & 92.55 & 92.56 & 92.94 \\
\hline Crude protein & 30.0 & 30.02 & 30.16 & 29.9 \\
\hline Ether extract & 6.65 & 6.71 & 6.47 & 6.72 \\
\hline Crude fiber & 2.15 & 2.37 & 2.20 & 2.28 \\
\hline Ash & 4.95 & 3.29 & 5.25 & 4.87 \\
\hline Carbohydrate & 55.73 & 57.60 & 55.90 & 56.21 \\
\hline \multicolumn{5}{|c|}{ Calculated energy value } \\
\hline Gross energy ${ }^{4}(\mathrm{Kcal} / \mathrm{Kg})$ & 479.51 & 486.14 & 476.83 & 479.35 \\
\hline Digestible energy (Kcal / Kg) & 4040 & 4100 & 4020 & 4040 \\
\hline Metabolize energy $(\mathrm{Kcal} / \mathrm{Kg})$ & 2610 & 2620 & 2590 & 2600 \\
\hline $\begin{array}{l}\text { Protein to energy ratio P /E ratio } \\
\mathrm{mg} \mathrm{CP} \mathrm{/} \mathrm{Kcal}\end{array}$ & 75.38 & 73.10 & 74.91 & 73.89 \\
\hline $\mathrm{CHO} / \mathrm{CP}$ ratio ${ }^{5}$ & 1.85 & 1.92 & 1.85 & 1.88 \\
\hline
\end{tabular}

1- Sunflower oil : cod liver oil with ratio of 1:1.

2-3- Minerals and vitamins premix composition is presented in Table 10.

4- Calculated by using factors of $5.65,9.4$ and 4.2 Kcal per gram of protein, fat and nitrogen free extract, respectively (NRC, 1993).

5- $\mathrm{CHO} / \mathrm{CP}$ ratio. Means carbohydrate to protein ratio. 
Table 8:Ingredients composition $(\mathrm{g} / \mathbf{1 0 0} \mathrm{g})$ and chemical analysis (\% dry matter basis) of the experimental diets used in the $2^{\text {nd }}$ additive (G).

\begin{tabular}{|c|c|c|c|c|}
\hline \multirow[t]{2}{*}{ Ingredients } & \multicolumn{4}{|c|}{ Garlen Allicin (G) levels } \\
\hline & 0 & G1 & G2 & G3 \\
\hline Fish meal & 10 & 10 & 10 & 10 \\
\hline Soybean meal & 44 & 44 & 44 & 44 \\
\hline Wheat milling by product & 15 & 15 & 15 & 15 \\
\hline Yellow corn & 21.38 & 21.38 & 21.38 & 21.38 \\
\hline Corn starch & 2.0 & 1.99 & 1.98 & 1.97 \\
\hline Oil mixture ${ }^{1}$ & 4.62 & 4.62 & 4.62 & 4.62 \\
\hline Vitamin premix $^{2}$ & 1.0 & 1.0 & 1.0 & 1.0 \\
\hline Mineral premix ${ }^{3}$ & 2.0 & 2.0 & 2.0 & 2.0 \\
\hline Garlen Allicin 25\% & 0.0 & 0.01 & 0.02 & 0.03 \\
\hline Total & 100 & 100 & 100 & 100 \\
\hline \multicolumn{5}{|c|}{ Chemical analysis } \\
\hline \begin{tabular}{|l|} 
Dry matter \\
\end{tabular} & 92.55 & 92.82 & 93.06 & 94.15 \\
\hline Crude protein & 30.0 & 29.97 & 30.03 & 29.86 \\
\hline Ether extract & 6.65 & 6.72 & 6.61 & 6.54 \\
\hline Crude fiber & 2.15 & 2.37 & 2.20 & 2.28 \\
\hline Ash & 4.95 & 5.33 & 4.26 & 5.5 \\
\hline Carbohydrate & 55.73 & 55.59 & 56.89 & 55.82 \\
\hline \multicolumn{5}{|c|}{ Calculated energy value } \\
\hline Gross energy ${ }^{4}(\mathrm{Kcal} / \mathrm{Kg})$ & 479.51 & 477.54 & 481.52 & 475.69 \\
\hline Digestible energy (Kcal / Kg) & 4040 & 4020 & 4070 & 4010 \\
\hline Metabolezable energy (Kcal /Kg) & 2610 & 2590 & 2610 & 2800 \\
\hline $\begin{array}{l}\text { Protein to energy ratio } \mathrm{P} / \mathrm{E} \text { ratio } \\
\mathrm{mg} \mathrm{CP} / \mathrm{Kcal}\end{array}$ & 75.38 & 73.11 & 73.80 & 74.40 \\
\hline $\mathrm{CHO} / \mathrm{CP}$ ratio ${ }^{5}$ & 1.85 & 1.85 & 1.89 & 1.87 \\
\hline
\end{tabular}

1- Sunflower oil : cod liver oil with ratio of 1:1.

2-3- Minerals and vitamins premix composition is presented in Table 10.

4- Calculated by using factors of $5.65,9.4$ and 4.2 Kcal per gram of protein, fat and nitrogen free extract, respectively (NRC, 1993).

5- $\mathrm{CHO} / \mathrm{CP}$ ratio. Means carbohydrate to protein ratio. 
Table 9: Ingredients composition ( $\mathrm{g} / 100 \mathrm{~g})$ and chemical analysis $(\%$ dry matter basis) of the experimental diets used in the $3^{\text {rd }}$ additive (XPC).

\begin{tabular}{|l|c|c|c|c|}
\hline \multirow{2}{*}{ Ingredients } & \multicolumn{4}{|c|}{ Diamond V (XPC) levels } \\
\cline { 2 - 5 } & $\mathbf{0}$ & XPC1 & XPC2 & XPC3 \\
\hline Fish meal & 10 & 10 & 10 & 10 \\
\hline Soybean meal & 44 & 44 & 44 & 44 \\
\hline Wheat milling by product & 15 & 15 & 15 & 15 \\
\hline Yellow corn & 21.38 & 21.38 & 21.38 & 21.38 \\
\hline Corn starch & 2.0 & 1.6 & 1.5 & 1.4 \\
\hline Oil mixture ${ }^{1}$ & 4.62 & 4.62 & 4.62 & 4.62 \\
\hline Vitamin premix ${ }^{2}$ & 1.0 & 1.0 & 1.0 & 1.0 \\
\hline Mineral premix ${ }^{3}$ & 2.0 & 2.0 & 2.0 & 2.0 \\
\hline XPC & 0.0 & 0.4 & 0.5 & 0.6 \\
\hline Total & 100 & 100 & 100 & 100 \\
\hline \multicolumn{4}{|c|}{ Chemical analysis } \\
\hline Dry matter & 92.55 & 93.37 & 92.90 & 93.27 \\
\hline Crude protein & 30.0 & 30.18 & 30.5 & 29.91 \\
\hline Ether extract & 6.65 & 6.33 & 6.40 & 6.56 \\
\hline Crude fiber & 2.15 & 2.37 & 2.20 & 2.28 \\
\hline Ash & 4.95 & 4.96 & 4.76 & 5.71 \\
\hline Carbohydrate & 55.73 & 56.15 & 56.11 & 55.53 \\
\hline \multicolumn{4}{|c|}{ Calculated energy value } \\
\hline Gross energy ${ }^{4}$ (Kcal / Kg) & 479.51 & 477.36 & 488.22 & 475.01 \\
\hline Digestible energy (Kcal / Kg) & 4040 & 4020 & 4040 & 4000 \\
\hline Metabolezable energy (Kcal /Kg) & 2610 & 2580 & 2600 & 2580 \\
\hline $\begin{array}{l}\text { Protein to energy ratio P /E ratio } \\
\text { mg CP / Kcal }\end{array}$ & 75.38 & 74.95 & 75.50 & 74.65 \\
\hline CHO / CP ratio ${ }^{5}$ & 1.85 & 1.86 & 1.84 & 1.85 \\
\hline
\end{tabular}

1- Sunflower oil : cod liver oil with ratio of 1:1.

2-3- Mineral and Vitamin premix composition is presented in Table 10.

4- Calculated by using factors of $5.65,9.4$ and $4.2 \mathrm{Kcal}$ per gram of protein, fat and nitrogen free extract, respectively (NRC, 1993).

5- $\mathrm{CHO} / \mathrm{CP}$ ratio. Means carbohydrate to protein ratio. 
Table 10: Composition $(/ \mathrm{kg})$ of vitamins and minerals premix* used in the experimental diets.

\begin{tabular}{|l|c|}
\hline Ingredient & Concentration \\
\hline Vitamin A & $15000 \mathrm{lu}$ \\
Vitamin $\mathrm{D}_{3}$ & $15000 \mathrm{lu}$ \\
Vitamin E & $2 \mathrm{mg}$ \\
Vitamin $\mathrm{K}_{3}$ & $2 \mathrm{mg}$ \\
Vitamin $\mathrm{B}_{1}$ & $2 \mathrm{mg}$ \\
Vitamin $\mathrm{B}_{2}$ & $2.5 \mathrm{mg}$ \\
Nicotine amide & $10 \mathrm{mg}$ \\
Vitamin $\mathrm{B}_{6}$ & $3 \mathrm{mg}$ \\
Vitamin $\mathrm{B}_{12}$ & $5 \mathrm{mcg}$ \\
Folic acid & $2 \mathrm{mg}$ \\
Ca - d -Pantothenate & $5.5 \mathrm{mg}$ \\
Calcium & $200 \mathrm{~g}$ \\
Phosphate & $90 \mathrm{~g}$ \\
Sodium & $40 \mathrm{~g}$ \\
Cupper & $2.5 \mathrm{~g}$ \\
Magnesium & $48 \mathrm{~g}$ \\
Manganese & $3.6 \mathrm{~g}$ \\
Zinc & $23.5 \mathrm{~g}$ \\
Iron & $8 \mathrm{~g}$ \\
Cobalt & $450 \mathrm{mg}$ \\
lodine & $200 \mathrm{mg}$ \\
Selenium & $20 \mathrm{mg}$ \\
\hline
\end{tabular}

${ }^{\star}$ Super Vit , Arab Veterinary Industrial Co. (Avico), Jordan.

*Eco Vit , Egyptian veterinary produced and feed a additives Co., Damiatta, Egypt.

\section{Experimental conditions:}

Fish were reared in fresh water. Temperature, $\mathrm{pH}$, dissolved oxygen and photoperiod values were $28 \pm 2{ }^{\circ} \mathrm{C}, 8.5 \pm 0.2,7 \mathrm{ppm}$ and $12 / 12$ hours light/darkness, respectively. Water exchange rate was $30 \%$ daily of the total volume of rearing water.

\section{Chemical analysis of diets:}

The tested diets were analyzed in triplicates. Chemical analysis was carried out according to the methods described by A.O.A.C. (1990) for dry matter, crude protein, ether extract, crude fiber and ash. Nitrogen free extract (carbohydrate) content was calculated by subtraction the total percentages of $\mathrm{CP}, \mathrm{EE}, \mathrm{CF}$ and ash from 100. The gross energy contents of the experimental diets and fish samples were calculated by using factors of 5.65 , 9.45 and $4.2 \mathrm{Kcal} / \mathrm{g}$ of protein, lipid and carbohydrate, respectively (NRC, 1993). Digestible energy content was calculated from standard physiological fuel values as 4,4 and $9 \mathrm{Kcal} / \mathrm{g}$ of protein, carbohydrate and lipid, respectively (Garling and Wilson, 1976). 
Measurements and calculations:

Gain, average daily gain (ADG), percentage average daily gain (ADG \%), and specific growth rate (SGR \%) were calculated according to the following equations:

Gain =final fish weith $(\mathrm{g})-$ initial fish weith $(\mathrm{g})$.

Gain $\%=$ Gain of fish $(\mathrm{g}) /$ initial weith of fish $(\mathrm{g}) \times 100$.

ADG = Gain (g) / time (day).

ADG \% $=\{A D G /$ Initial weight of fish $(\mathrm{g})\} \times 100$.

SGR $=100 X\left\{\left(\ln W_{2}-\ln W_{1}\right) / T\right\}$

Where............ $W_{2}$ is the final weight of fish $(G)$.

Where............ $W_{1}$ is the initial weight of fish $(G)$.

In: is natural log.

$\mathrm{T}$ : is the period (day).

Condition factor $(\mathbf{K})=\left\{(\right.$ Final weight $) /\left(\right.$ Final Length) $\left.{ }^{3}\right\} \times 100$

Cost - benefit analysis:

Cost - benefit analysis was used to express the economic analysis due to the cost of feed and their profitability according to New (1987).

Incident cost (IC):

Incident cost is expressing the cost of feed consumed to produce one kilogram fresh fish. IC = cost of feed consumed / kg fish produced.

Profit index (PI):

Profit index $(\mathrm{PI})$ is expressed as the value of fish produced divided cost of feed.

$\mathbf{P I}=$ Price of fish produced $/$ price of feed consumed.

The prices in LE/Kg (year 2012) for fish 7.000, control diet 4.087, Aqua

$0.01 \%$ diet 4.096 , Aqua $0.02 \%$ diet 4.0105 , Aqua $0.03 \%$ diet 4.114 , Garlen

$0.01 \%$ diet 4.099 , Garlen $0.02 \%$ diet 4.112 , Garlen $0.03 \%$ diet 4.124 , XPC

$0.4 \%$ diet 4.147 , XPC $0.5 \%$ diet 4.162 , and XPC $0.6 \%$ diet 4.177 .

Statistical analysis:

Data obtained were analyzed using one-way analysis of variance. All statistical analysis were performed according to SAS (2006). Differences were subjected to Duncan's (1955). Multiple Range - test.

\section{RESULTS AND DISCUSSION}

\section{Growth performance:}

Gain and survival:

The next tables present the mean values of growth performance parameters of the experimented tilapia fish fed the Aqua Superzyme containing experimental diets (Table 11), the Garlen Allicin probiotic containing experimental diets (Table 12), and the Diamond V (Original XPC) probiotic containing experimental diets (Table 13), concerning initial bodyweight (IW), final bodyweight (FW), total weifgt gain (TWG), average daily gain (ADG), relative growth rate (RGR), specific growth rate (SGR), and survival rate $(\mathrm{SR})$.

The data cleared that all of the pre- and probiotics used in the present study led to significant $(P \leq 0.05)$ improvements in all tested criteria 
comparing with their controls (without additives); although, there were no significant $(P \geq 0.05)$ differences among the initial body weights. $A_{1}, G_{1}$, and $\mathrm{XPC}_{2}$ were the best treatments, comparing with their other levels (Tables 11, 12 , and 13 , respectively).

Table 11:Growth performance of Nile tilapia (O. niloticus) fish fed the Aqua Superzyme containing experimental diets.

\begin{tabular}{|c|c|c|c|c|c|c|c|}
\hline Treat. & IW (g) & $\mathrm{FW}(\mathrm{g})$ & TWG (g) & $\begin{array}{l}\text { ADG } \\
\text { (mg) }\end{array}$ & $\begin{array}{l}\text { RGR } \\
(\%)\end{array}$ & $\begin{array}{l}\text { SGR } \\
(\% / d)\end{array}$ & SR(\%) \\
\hline Cont. & \begin{tabular}{|l|}
10.90 \\
\end{tabular} & $39.86^{c}$ & $28.96^{c}$ & $258.57^{c}$ & $266.16^{c}$ & $1.15^{\mathrm{c}}$ & $85.49^{b}$ \\
\hline$\overline{A_{1}}$ & \begin{tabular}{|l|}
10.79 \\
\end{tabular} & $52.81^{a}$ & $42.02^{\mathrm{a}}$ & $375.18^{a}$ & $389.20^{a}$ & $1.41^{a}$ & $95.95^{a}$ \\
\hline$A_{2}$ & 10.95 & $46.81^{b}$ & $35.85^{b}$ & $320.15^{b}$ & $327.33^{b}$ & $1.29^{b}$ & $94.33^{a}$ \\
\hline$A_{3}$ & \begin{tabular}{|l|}
10.45 \\
\end{tabular} & $45.39^{b}$ & $34.94^{b}$ & $312.02^{b}$ & $334.40^{b}$ & $1.31^{\mathrm{b}}$ & $89.16^{\mathrm{ab}}$ \\
\hline$P>F$ & \begin{tabular}{|l|}
0.106 \\
\end{tabular} & 0.0001 & 0.0002 & 0.0002 & 0.0005 & 0.0006 & 0.021 \\
\hline \pm SE & 0.135 & 1.01 & 1.04 & 9.31 & 11.38 & 0.025 & 2.0 \\
\hline
\end{tabular}

Table 12:Growth performance of Nile tilapia (O. niloticus) fed the Garlen Allicin probiotic containing experimental diets.

\begin{tabular}{|c|c|c|c|c|c|c|c|}
\hline Treat. & IW (g) & $F W(g)$ & TWG (g) & $\begin{array}{l}\text { ADG } \\
(\mathrm{mg})\end{array}$ & $\begin{array}{c}\text { RGR } \\
\text { (\%) }\end{array}$ & $\begin{array}{l}\text { SGR } \\
\text { (\%/d) }\end{array}$ & SR(\%) \\
\hline Cont & .90 & $39.86^{d}$ & $28.96^{d}$ & $258.57^{d}$ & $266.16^{d}$ & $1.15^{\mathrm{d}}$ & $85.49^{b}$ \\
\hline $\mathrm{G}_{1}$ & 10.86 & $62.43^{a}$ & $51.56^{a}$ & $460.41^{a}$ & $474.66^{a}$ & $1.56^{a}$ & $97.77^{a}$ \\
\hline $\mathrm{G}_{2}$ & 11.05 & $52.27^{b}$ & $41.21^{b}$ & $368.00^{b}$ & $372.83^{b}$ & $1.39^{b}$ & $95.83^{\mathrm{ab}}$ \\
\hline $\mathrm{G}_{3}$ & 11.08 & $47.25^{c}$ & $36.16^{c}$ & $322.89^{c}$ & $326.30^{c}$ & $1.29^{c}$ & $93.59^{\mathrm{ab}}$ \\
\hline$P>F$ & 0.733 & 0.0001 & 0.0001 & \begin{tabular}{|l|}
0.0001 \\
\end{tabular} & 0.0001 & 0.0001 & 0.124 \\
\hline \pm SE & 0.166 & 0.91 & 0.89 & 8.01 & 9.24 & 0.02 & 3.34 \\
\hline
\end{tabular}

Table 13:Growth performance of Nile tilapia (O. niloticus) fed the Diamond V (Original XPC) probiotic, containing experimental diets.

\begin{tabular}{|l|c|c|c|c|c|c|c|}
\hline Treat. & IW (g) & FW(g) & TWG (g) & $\begin{array}{c}\text { ADG } \\
(\mathbf{m g})\end{array}$ & $\begin{array}{c}\text { RGR } \\
(\%)\end{array}$ & $\begin{array}{c}\text { SGR } \\
(\% / d)\end{array}$ & SR(\%) \\
\hline Cont. & 10.90 & $39.86^{\mathrm{d}}$ & $28.96^{\mathrm{c}}$ & $258.57^{\mathrm{c}}$ & $266.16^{\mathrm{c}}$ & $1.15^{\mathrm{c}}$ & $85.49^{\mathrm{b}}$ \\
\hline $\mathrm{XPC}_{1}$ & 10.79 & $67.21^{\mathrm{b}}$ & $56.42^{\mathrm{b}}$ & $503.75^{\mathrm{b}}$ & $522.93^{\mathrm{b}}$ & $1.63^{\mathrm{b}}$ & $93.75^{\mathrm{a}}$ \\
\hline $\mathrm{XPC}_{2}$ & 10.95 & $75.35^{\mathrm{a}}$ & $64.39^{\mathrm{a}}$ & $574.97^{\mathrm{a}}$ & $587.86^{\mathrm{a}}$ & $1.72^{\mathrm{a}}$ & $100^{\mathrm{a}}$ \\
\hline $\mathrm{XPC}_{3}$ & 10.45 & $63.13^{\mathrm{c}}$ & $52.68^{\mathrm{b}}$ & $470.41^{\mathrm{b}}$ & $504.23^{\mathrm{b}}$ & $1.60^{\mathrm{b}}$ & $97.77^{\mathrm{a}}$ \\
\hline $\mathrm{P}>\mathrm{F}$ & 0.106 & 0.0001 & 0.0001 & 0.0001 & 0.0001 & 0.0001 & 0.016 \\
\hline$\pm \mathrm{SE}$ & 0.13 & 1.17 & 1.25 & 11.18 & 15.21 & 0.025 & 2.52 \\
\hline
\end{tabular}

a-c: means in the same column having different letters are significantly $(P \leq 0.05)$ different. 
Table 14 presentes the comparison effect of the three treatments (additives) and three addition levels (besides the control). The best FW $(75.35 \mathrm{~g})$, TWG $(64.39 \mathrm{~g})$, RGR (587.86 \%), SGR (1.72 \%/d), and SR $(100 \%)$ was $3^{\star} 2\left(\mathrm{XPC}_{2}\right.$, the probiotic Diamond V- Original XPC at $0.5 \%$ of the diet).

Table 14: Comparison (treatment, $T$ * level, $L$ ) of growth performance of Nile tilapia fish (O. niloticus) fed the Aqua Superzyme, Garlen Allicin, and Diamond V (Original XPC) containing experimental diets.

\begin{tabular}{|l|c|c|c|c|c|c|c|}
\hline $\mathbf{T}^{*} \mathbf{L}$ & $\mathbf{I W}(\mathbf{g})$ & $\mathbf{F W}(\mathbf{g})$ & $\begin{array}{c}\text { TWG } \\
(\mathbf{g})\end{array}$ & $\begin{array}{c}\text { ADG } \\
(\mathbf{m g})\end{array}$ & $\begin{array}{c}\text { RGR } \\
(\%)\end{array}$ & $\begin{array}{c}\text { SGR } \\
(\% / \mathbf{d})\end{array}$ & $\mathbf{S R}(\%)$ \\
\hline Cont. & 10.90 & $39.86^{\mathrm{f}}$ & $28.96^{\mathrm{f}}$ & $258.57^{\mathrm{f}}$ & $266.16^{\mathrm{f}}$ & $1.15^{\mathrm{f}}$ & $85.49^{\mathrm{c}}$ \\
\hline $1^{*} 1$ & 10.79 & $52.81^{\mathrm{d}}$ & $42.02^{\mathrm{d}}$ & $375.18^{\mathrm{d}}$ & $389.20^{\mathrm{d}}$ & $1.41^{\mathrm{d}}$ & $95.95^{\mathrm{ab}}$ \\
\hline $1^{*} 2$ & 10.95 & $46.81^{\mathrm{e}}$ & $35.85^{\mathrm{e}}$ & $320.15^{\mathrm{e}}$ & $327.33^{\mathrm{e}}$ & $1.29^{\mathrm{e}}$ & $94.33^{\mathrm{ab}}$ \\
\hline $1^{*} 3$ & 10.45 & $45.39^{\mathrm{e}}$ & $34.94^{\mathrm{e}}$ & $312.02^{\mathrm{e}}$ & $334.40^{\mathrm{e}}$ & $1.31^{\mathrm{e}}$ & $89.16^{\mathrm{bc}}$ \\
\hline $2^{*} 1$ & 10.86 & $62.43^{\mathrm{c}}$ & $51.56^{\mathrm{c}}$ & $460.41^{\mathrm{c}}$ & $476.66^{\mathrm{c}}$ & $1.56^{\mathrm{c}}$ & $97.77^{\mathrm{ab}}$ \\
\hline $2^{\star} 2$ & 11.05 & $52.27^{\mathrm{d}}$ & $41.21^{\mathrm{d}}$ & $368.0^{\mathrm{d}}$ & $372.83^{\mathrm{d}}$ & $1.39^{\mathrm{d}}$ & $95.83^{\mathrm{ab}}$ \\
\hline $2^{*} 3$ & 11.08 & $47.52^{\mathrm{e}}$ & $36.16^{\mathrm{e}}$ & $322.89^{\mathrm{e}}$ & $326.30^{\mathrm{e}}$ & $1.29^{\mathrm{e}}$ & $93.59^{\mathrm{abc}}$ \\
\hline $3^{*} 1$ & 10.79 & $67.21^{\mathrm{b}}$ & $56.42^{\mathrm{b}}$ & $503.75^{\mathrm{b}}$ & $522.93^{\mathrm{b}}$ & $1.63^{\mathrm{b}}$ & $93.75^{\mathrm{abc}}$ \\
\hline $3^{*} 2$ & 10.95 & $75.35^{\mathrm{a}}$ & $64.39^{\mathrm{a}}$ & $574.97^{\mathrm{a}}$ & $587.86^{\mathrm{a}}$ & $1.72^{\mathrm{a}}$ & $100^{\mathrm{a}}$ \\
\hline $3^{*} 3$ & 10.45 & $63.13^{\mathrm{c}}$ & $52.68^{\mathrm{c}}$ & $470.41^{\mathrm{c}}$ & $504.23^{\mathrm{bc}}$ & $1.60^{\mathrm{bc}}$ & $97.77^{\mathrm{ab}}$ \\
\hline $\mathrm{P}>\mathrm{F}$ & 0.0082 & 0.0001 & 0.0001 & 0.0001 & 0.0001 & 0.0001 & 0.0362 \\
\hline$\pm \mathrm{SE}$ & 0.117 & 1.07 & 1.089 & 9.731 & 11.724 & 0.0214 & 2.668 \\
\hline
\end{tabular}

a-f: means in the same column having different letters are significantly $(P \leq 0.05)$ different

Amer (2012) concluded that Diamond $V X P$ (inactive yeast a commercial product containing $100 \%$ dried Saccharomyces Cerevisae distributed by DIAMOND V mils, Cidar Rapids, IOWA, and USA) could be used successfully as feed additive for feeding Nile tilapia Oreochromis niloticus without any adverse effects on their productive performance. Generally, it improves growth performance than control diet. Also, results obtained showed that using Diamond $V X P$ at level $(1.5 \mathrm{~g})$ had the best results on productive with levels of protein (30\%). Moreover, it could be suggested that dietary supplement with Diamond $V X P(1.5 \mathrm{~g})$ is useful in the intensive production system of fish.

Hassan (2013) obtained results concerning the effects of the probiotic Hydroyeast Aquaculture ${ }^{\circledR}$ on adult male O. niloticus showed that the $15 \mathrm{~g} / \mathrm{kg}$ diet realized best significantly $(P \leq 0.05)$ values for final body weight, average weight gain, average daily gain, relative growth rate, and specific growth rate. However, about adult females' $O$. niloticus, the $10 \mathrm{~g}$ probiotic $/ \mathrm{kg}$ diet was the best treatment concerning the significantly $(P \leq 0.05)$ improvement of the growth performance parameters (final body weight, AWG, RGR, ADG and SGR). So, based on the obtained results, the optimum level of the tested probiotic Hydroyeast Aquaculture ${ }^{\circledR}$ was depinding on fish sex. 


\section{Condition factor:}

Data of the condition factor $(K)$ for the experimented fish fed the Aqua Superzyme containing experimental diets (Table 15), the Garlen Allicin probiotic containing experimental diets (Table 16), and the Diamond $V$ (Original XPC) probiotic containing experimental diets (Table 17) were calculated on the bases of standard and total lengths, respectively, It revealed that the significantly $(P \leq 0.05)$ highest level among the tested levels of each additive was of $A_{1}, G_{1}$, and XPC 1 for both $K_{1}$ and $K_{2}$. However, all additives used raised the condition factor comparing with their controls, but Diamond $\mathrm{V}$ (Original XPC) was responsible for the highest $\mathrm{k}$-factors comparing with the other additives.

Table 15:Condition factor of Nile tilapia (O. niloticus) fed the Aqua Superzyme prebiotic of the experimental diets.

\begin{tabular}{|l|c|c|}
\hline Treat & $\mathbf{K}_{\mathbf{1}}$ (standard length basis) & $\mathbf{K}_{\mathbf{2}}$ (total length basis) \\
\hline Control & $5.40^{\mathrm{b}}$ & $2.91^{\mathrm{b}}$ \\
\hline $\mathrm{A}_{1}$ & $8.13^{\mathrm{a}}$ & $4.39^{\mathrm{a}}$ \\
\hline $\mathrm{A}_{2}$ & $6.94^{\mathrm{ab}}$ & $3.89^{\mathrm{a}}$ \\
\hline $\mathrm{A}_{3}$ & $6.60^{\mathrm{ab}}$ & $3.52^{\mathrm{ab}}$ \\
\hline $\mathrm{P}>\mathrm{F}$ & 0.0723 & 0.0297 \\
\hline$\pm \mathrm{SE}$ & 0.605 & 0.277 \\
\hline
\end{tabular}

a-b: means in the same column having different letters are significantly $(P \leq 0.05)$ different.

Table 16:Condition factor of Nile tilapia (O. niloticus) fed the Garlen Allicin probiotic of the experimental diets.

\begin{tabular}{|l|l|l|}
\hline Treat & $\mathrm{K}_{1}$ (standard length basis ) & $\mathrm{K}_{2}$ (total length basis) \\
\hline Control & $5.40^{\mathrm{b}}$ & $2.91^{\mathrm{b}}$ \\
\hline $\mathrm{G}_{1}$ & $8.09^{\mathrm{a}}$ & $4.47^{\mathrm{a}}$ \\
\hline $\mathrm{G}_{2}$ & $6.58^{\mathrm{ab}}$ & $3.69^{\mathrm{ab}}$ \\
\hline $\mathrm{G}_{3}$ & $6.27^{\mathrm{ab}}$ & $3.65^{\mathrm{ab}}$ \\
\hline $\mathrm{P}>\mathrm{F}$ & 0.0488 & 0.0588 \\
\hline$\pm \mathrm{SE}$ & 0.553 & 0.326 \\
\hline
\end{tabular}

a-b: means in the same column having different letters are significantly $(P \leq 0.05)$ different.

Table 17: Condition factor of Nile tilapia (O. niloticus) fed the Diamond V (Original XPC) probiotic of the experimental diets.

\begin{tabular}{|c|c|c|}
\hline Treat & $\mathbf{K}_{\mathbf{1}}$ (standard length basis) & $\mathbf{K}_{\mathbf{2}}$ (total length basis) \\
\hline Control & $5.40^{\mathrm{b}}$ & $2.91^{\mathrm{b}}$ \\
\hline $\mathrm{XPC}_{1}$ & $10.08^{\mathrm{a}}$ & $5.29^{\mathrm{a}}$ \\
\hline $\mathrm{XPC}_{2}$ & $9.74^{\mathrm{a}}$ & $5.33^{\mathrm{a}}$ \\
\hline $\mathrm{XPC}_{3}$ & $8.82^{\mathrm{a}}$ & $4.75^{\mathrm{a}}$ \\
\hline $\mathrm{P}>\mathrm{F}$ & 0.0009 & 0.0003 \\
\hline$\pm \mathrm{SE}$ & 0.528 & 0.326 \\
\hline
\end{tabular}
different. 
Table 18 illustrates the comparison data of K-factor, it showes that the highest K-factor based on total lengths was caculated with the treatment * level $3{ }^{*} 2\left(\mathrm{XPC}_{2}\right.$, Diamond $\mathrm{V}$, Original at inclusion level $0.5 \%$ of the diet).

Table 18: Comparison among the three additives and their inclusion levels ( 3 plus control) for condition factor of Nile tilapia ( $O$. niloticus) fed the Aqua Superzyme, Garlen Allicin, and Diamond V (Original XPC) including experimental diets.

\begin{tabular}{|l|c|c|}
\hline Treat & $\mathbf{K}_{\mathbf{1}}$ (Standard length) & $\mathbf{K}_{\mathbf{2}}$ (Total length) \\
\hline control & $5.40^{\mathrm{e}}$ & $2.91^{\mathrm{d}}$ \\
\hline $1^{\star} 1$ & $8.13^{\mathrm{bcd}}$ & $4.39^{\mathrm{abc}}$ \\
\hline $1^{\star} 2$ & $6.94^{\mathrm{cde}}$ & $3.89^{\mathrm{bc}}$ \\
\hline $1^{\star} 3$ & $6.60^{\mathrm{de}}$ & $3.52^{\mathrm{cd}}$ \\
\hline $2^{\star} 1$ & $8.09^{\mathrm{bcd}}$ & $4.47^{\mathrm{abc}}$ \\
\hline $2^{\star} 2$ & $6.58^{\mathrm{de}}$ & $3.69^{\mathrm{cd}}$ \\
\hline $2^{\star} 3$ & $6.27^{\mathrm{de}}$ & $3.65^{\mathrm{cd}}$ \\
\hline $3^{\star} 1$ & $10.08^{\mathrm{a}}$ & $5.29^{\mathrm{a}}$ \\
\hline $3^{\star} 2$ & $9.74^{\mathrm{ab}}$ & $5.33^{\mathrm{a}}$ \\
\hline $3^{\star} 3$ & $8.82^{\mathrm{abc}}$ & $4.75^{\mathrm{ab}}$ \\
\hline $\mathrm{P}>\mathrm{F}$ & 0.0002 & 0.0001 \\
\hline$\pm \mathrm{SE}$ & 0.589 & 0.294 \\
\hline
\end{tabular}
a-e: means in the same column having different letters are significantly $(P \leq 0.05)$ different.

Lara-Flores et al. (2003) evaluated the effects of probiotics on growth performance in Nile tilapia under two stress factors. These stressors were dietary protein level and stocking density. The probiotics used based either upon Streptococcus faecium and Lactobacillus acidophilus or Saccharomyces cerevisiae. Results indicated that the fry fed diets with a probiotics supplement exhibited greater growth than those led the control diet without probiotic. Of all probiotic treatments, yeast was an appropriate growth stimulating additive in tilapia cultivation. The authors stated that the increased growth rate as a positive response to added probiotics could be attributed to increased efficiency of existing digestive processes or by promoting the digestion of previously indigestible substances. In addition, probiotics may have a protein sparing effect.

Two strains of Saccharomyces cerevisiae were tested as probiotics for rainbow trout fry, during the first month of feeding. Each strain was introduced into separate diets, at the rate of $10^{6} \mathrm{CFU} \mathrm{g}^{-1}$ and their effects were compared with those of a control diet. In all groups, the counts of bacteria associated with trout intestine were maximum 10 days post start feeding (dpsf; $10^{7}$ CFU $\mathrm{g}^{-1}$ ). An autochthonous yeast, Debaryomyces hansenii, was also retrieved associated to the intestine of the control group in high numbers after 240 degree days of experiment $\left(10^{4}-10^{5} \mathrm{CFU} \mathrm{g}{ }^{-1}\right)$, while the colonization level was significantly less in trout fed the probiotic diets. The effect of the dietary yeast was observed by assaying the activity of three enzymes in the brush border membrane of the enterocytes: alkaline 
phosphatase (AP), Y-glutamyl-transpeptidase (GGT), and leucine-aminopeptidase N (LAP). Both $S$. boulardii and $D$. hansenii seemed to stimulate digestive maturation in fish, but the natural colonization by $D$. hansenii was likely too late for trout reared at optimal temperature. The supplementation of trout starter diet with $S$. boulardii may be particularly useful in fast growing conditions (Waché et al., 2006).

In an in door experiment, the prebiotic Avian Plus was evaluated by adding it at graded levels into isonitrogenous-isocaloric-diets of tilapia fish for 112 days. The obtained results revealed the superiority of the Avian Plus containing diets, particularly at level of $25 \mathrm{mg} / \mathrm{kg}$ feed which led to significantly $(P \leq 0.05)$ best final bodyweight, average bodyweight gain, average daily bodyweight gain, relative growth rate, and specific growth rate comparing with the control (Abdelhamid et al., 2011).

Two experiments were carried out. In both experiments, Nile tilapia fry $(0.1 \mathrm{~g}$ initial bodyweight) were stocked into glass aquaria at 100 fry / aquarium in duplicate aquaria / treatment. The experimental diets were offered daily at a feeding rate of $10 \%$ of the fish biomass for 21 days (hormone-treated diets as commercially done for sex reverse) followed by 8 or 12 weeks as experimental periods in the $1^{\text {st }}$ and the $2^{\text {nd }}$ experiments, respectively. Bio-Mos ${ }^{\circledR}$ and T-Protphyt 2000 were added to the $1^{\text {st }}$ and the $2^{\text {nd }}$ experiments, respectively at graded levels. The results obtained revealed no significant $(P \geq 0.05)$ differences among treatments in both experiments concerning growth performance. However, the diet containing testosterone alone in the $1^{\text {st }}$ experiment (T4) and that containing T-Protphyt 2000 at $4 \mathrm{~g} /$ $\mathrm{Kg}$ diet in the $2^{\text {nd }}$ experiment reflected the best growth performance. The dietary inclusion of Bio-Mos ${ }^{\circledR}$ was not beneficial, whereas the dietary inclusion of T-Protphyt 2000 at $4 \mathrm{~g} / \mathrm{Kg}$ diet was positively effective in increasing the growth performance of the tilapia fry (Abdelhamid et al., 2012).

Amer (2012) concluded that Diamond $V X P$ (inactive yeast a commercial product containing $100 \%$ dried Saccharomyces cerevisae distributed by DIAMOND V mils, Cidar Rapids, IOWA, and USA) could be used successfully as feed additive for feeding Nile tilapia Oreochromis niloticus without any adverse effects on their productive performance. Generally, it improves feed utilization than control diet. Also, results obtained showed that using Diamond $V X P$ at level $(1.5 \mathrm{~g})$ had the best results on productive with levels of protein (30\%). Moreover, it could be suggested that dietary supplement with Diamond $V X P(1.5 \mathrm{~g})$ is useful in the intensive production system of fish.

Hassan (2013) obtained results concerning the effects of the probiotic Hydroyeast Aquaculture ${ }^{\circledR}$ on adult male $O$. niloticus showed that the $15 \mathrm{~g} / \mathrm{kg}$ diet realized best significantly $(P \leq 0.05)$ values for growth performance.

\section{Economics:}

Economically, A1, G1 and XPC2 (Tables 19, 20, and 21, respectively) were the best levels among the tested concentrations for the different substances examined in the present study. The interaction $\left(T^{*} \mathrm{~L}\right)$ 
effect on the economic efficiency of feeding Nile tilapia fish the experimental diets included Aqua Superzyme, Garlen Allicin, and Diamond V-Original (T) with their different levels $(\mathrm{L})$ is given in Table 22. From which it is clear that the best economic diets are $3^{\star} 2$ (Diamond V-Original at $0.5 \%$ of the diet).

Table 19: Effect of Nile tilapia (O. niloticus) fed the Aqua Superzyme prebiptic supplementation on the economic efficiency of the experimental diets.

\begin{tabular}{|c|c|c|c|c|c|}
\hline Treat & $\begin{array}{c}\text { Total }^{1} \\
\text { output }^{1}\end{array}$ & In put ${ }^{2}$ & Net return ${ }^{3}$ & $\begin{array}{c}\text { Economic } \\
\text { efficiency } \%\end{array}$ & $\begin{array}{c}\text { Relative } \\
\text { economic } \\
\text { efficiency }\end{array}$ \\
\hline Control & $0.87^{c}$ & $0.25^{c}$ & $0.62^{c}$ & $248.10^{c}$ & $100.0^{b}$ \\
\hline$A_{1}$ & $1.26^{a}$ & $0.31^{a}$ & $0.95^{a}$ & $307.86^{a}$ & $124.40^{a}$ \\
\hline$A_{2}$ & $1.07^{b}$ & $0.28^{b}$ & $0.78^{b}$ & $275.80^{b}$ & $111.33^{b}$ \\
\hline$A_{3}$ & $1.05^{b}$ & $0.28^{b}$ & $0.76^{b}$ & $265.10^{b c}$ & $107.0^{b}$ \\
\hline$P>F$ & 0.002 & 0.0001 & 0.0002 & 0.004 & 0.010 \\
\hline \pm SE & 0.031 & 0.004 & 0.028 & 7.76 & 3.76 \\
\hline
\end{tabular}

a-c: means in the same column having different letters are significantly $(P \leq 0.05)$ different.

Total feed costs per treatment (LE/kg diet) = Feed costs per one $\mathbf{k g}$ diet $\mathbf{x}$ feed intake

1-Total outputs per treatment $(\mathrm{LE} / \mathrm{kg})=$ fish price $x$ total fish production

2- Total fish production per treatment $=$ final number of fish $x$ fish weight gain

3- Net return per treatment $(L E)=$ total outputs - total feed costs

4- Economic efficiency per treatment(\%) = (net return / total feed costs) $\times 100$

5- Relative economic efficiency relative to the control (100).

Table 20: Effect of Nile tilapia (O. niloticus) fed the Garlen Allicin probiptic supplementation on the economic efficiency of the experimental diets.

\begin{tabular}{|c|c|c|c|c|c|}
\hline Treat & $\begin{array}{l}\text { Total } \\
\text { output }\end{array}$ & In put & Net return & $\begin{array}{c}\text { Economic } \\
\text { Efficiency, \% }\end{array}$ & $\begin{array}{c}\text { Relative } \\
\text { economic } \\
\text { efficiency }\end{array}$ \\
\hline Control & $0.87^{d}$ & $0.25^{c}$ & $0.62^{d}$ & $248.10^{c}$ & $100.0^{c}$ \\
\hline $\mathrm{G}_{1}$ & $1.54^{\mathrm{a}}$ & $0.33^{a}$ & $1.21^{\mathrm{a}}$ & $359.11^{a}$ & $144.66^{a}$ \\
\hline $\mathrm{G}_{2}$ & $1.23^{b}$ & $0.32^{b}$ & $0.91^{\mathrm{b}}$ & $284.25^{b}$ & $114.66^{b}$ \\
\hline $\mathrm{G}_{3}$ & $1.085^{c}$ & $0.31^{b}$ & $0.77^{c}$ & $247.72^{c}$ & $100^{c}$ \\
\hline$P>F$ & 0.0001 & 0.0001 & 0.0001 & 0.0001 & 0.0001 \\
\hline $\pm S E$ & 0.026 & 0.004 & 0.026 & 9.486 & 2.09 \\
\hline
\end{tabular}

a-d: means in the same column having different letters are significantly $(P \leq 0.05)$ different. 
Table 21:Effect of Nile tilapia (O. niloticus) fed the Diamond V (Original XPC) probiotic supplementation on the economic efficiency of the experimental diets.

\begin{tabular}{|l|c|c|c|c|c|}
\hline Treat & $\begin{array}{c}\text { Total } \\
\text { output }\end{array}$ & In put & Net return & $\begin{array}{c}\text { Economic } \\
\text { Efficiency, \% }\end{array}$ & $\begin{array}{c}\text { Relative } \\
\text { economic } \\
\text { efficiency }\end{array}$ \\
\hline Control & $0.87^{\mathrm{c}}$ & $0.25^{\mathrm{d}}$ & $0.62^{\mathrm{c}}$ & $248.10^{\mathrm{b}}$ & $100.0^{\mathrm{b}}$ \\
\hline $\mathrm{XPC}_{1}$ & $1.69^{\mathrm{b}}$ & $0.37^{\mathrm{b}}$ & $1.32^{\mathrm{b}}$ & $355.74^{\mathrm{a}}$ & $143.66^{\mathrm{a}}$ \\
\hline $\mathrm{XPC}_{2}$ & $1.93^{\mathrm{a}}$ & $0.41^{\mathrm{a}}$ & $1.52^{\mathrm{a}}$ & $368.05^{\mathrm{a}}$ & $148.66^{\mathrm{a}}$ \\
\hline $\mathrm{XPC}_{3}$ & $1.58^{\mathrm{b}}$ & $0.34^{\mathrm{c}}$ & $1.23^{\mathrm{b}}$ & $356.0^{\mathrm{a}}$ & $143.66^{\mathrm{a}}$ \\
\hline $\mathrm{P}>\mathrm{F}$ & 0.0001 & 0.0001 & 0.0001 & 0.0001 & 0.0004 \\
\hline$\pm \mathrm{SE}$ & 0.037 & 0.005 & 0.033 & 7.41 & 5.01 \\
\hline
\end{tabular}

a-d: means in the same column having different letters are significantly $(P \leq 0.05)$ different.

Table 22:Comparison effect of feeding Aqua Superzyme, Garlen Allicin, and Diamond V-Original supplementations $(T)$ with their different levels (L) on the economic efficiency of the experimental diets fet to Nile tilapia (O. niloticus).

\begin{tabular}{|l|c|c|c|c|c|}
\hline$T^{\star} \mathbf{L}$ & $\begin{array}{l}\text { Total } \\
\text { output }\end{array}$ & In put & Net return & $\begin{array}{l}\text { Economic } \\
\text { Efficiency, \% }\end{array}$ & $\begin{array}{l}\text { Relative } \\
\text { economic } \\
\text { efficiency }\end{array}$ \\
\hline Control & $0.868^{\mathrm{f}}$ & $0.249^{\mathrm{f}}$ & $0.619^{\mathrm{f}}$ & $248.10^{\mathrm{d}}$ & $100.0^{\mathrm{d}}$ \\
\hline $1^{\star} 1$ & $1.26^{\mathrm{d}}$ & $0.309^{\mathrm{d}}$ & $0.951^{\mathrm{d}}$ & $307.86^{\mathrm{b}}$ & $124.40^{\mathrm{b}}$ \\
\hline $1^{\star 2}$ & $1.075^{\mathrm{e}}$ & $0.286^{\mathrm{e}}$ & $0.789^{\mathrm{e}}$ & $275.80^{\mathrm{c}}$ & $111.33^{\mathrm{cd}}$ \\
\hline $1^{\star} 3$ & $1.048^{\mathrm{e}}$ & $0.287^{\mathrm{e}}$ & $0.761^{\mathrm{e}}$ & $265.10^{\text {cd }}$ & $107.0^{\mathrm{cd}}$ \\
\hline $2^{\star} 1$ & $1.547^{\mathrm{c}}$ & $0.337^{\mathrm{c}}$ & $1.209^{\mathrm{c}}$ & $359.11^{\mathrm{a}}$ & $144.66^{\mathrm{a}}$ \\
\hline $2^{\star} 2$ & $1.236^{\mathrm{d}}$ & $0322^{\mathrm{d}}$ & $0.915^{\mathrm{d}}$ & $284.25^{\mathrm{bc}}$ & $114.66^{\mathrm{bc}}$ \\
\hline $2^{\star} 3$ & $1.085^{\mathrm{e}}$ & $0.312^{\mathrm{d}}$ & $0.773^{\mathrm{e}}$ & $247.72^{\mathrm{d}}$ & $100.0^{\mathrm{d}}$ \\
\hline $3^{\star} 1$ & $1.692^{\mathrm{b}}$ & $0.371^{\mathrm{b}}$ & $1.321^{\mathrm{b}}$ & $355.74^{\mathrm{a}}$ & $143.66^{\mathrm{a}}$ \\
\hline $3^{\star} 2$ & $1.932^{\mathrm{a}}$ & $0.413^{\mathrm{a}}$ & $1.519^{\mathrm{a}}$ & $368.05^{\mathrm{a}}$ & $148.66^{\mathrm{a}}$ \\
\hline $3^{\star} 3$ & $1.580^{\mathrm{c}}$ & $0.346^{\mathrm{c}}$ & $1.234^{\mathrm{bc}}$ & $356.0^{\mathrm{a}}$ & $143.66^{\mathrm{a}}$ \\
\hline $\mathrm{P}>\mathrm{F}$ & 0.0001 & 0.0001 & 0.0001 & 0.0001 & 0.0001 \\
\hline$\pm \mathrm{SE}$ & 0.0326 & 0.0049 & 0.030 & 8.3418 & 4.1791 \\
\hline
\end{tabular}

a-f: means in the same column having different letters are significantly $(P \leq 0.05)$ different

El-Haroun et al. (2006) and El-Haroun (2007) reported that the diets containig probiotic Biogen ${ }^{\circledR}$ realised significantly $(P \leq 0.01)$ the highest net return and the lowest total cost by Nile tilapia and African catfish, respectively comparing with the control diets. Mohamed et al. (2007) also reported that dietary inclusion of Biogen ${ }^{\circledR}$ improved the economic evaluation for Nile tilapia production.

Egyptian populations of African catfish Clarias gariepinus $(24.34 \pm 1.38 \mathrm{~g})$ were fed a formulated diet containing approximately $31 \%$ (analyzed value) crude protein and cultured for 214 days. Four levels of dried brewer's yeast, Saccharomyces cerevisiae, (DY) were used as feed additives at levels of $0.0,1.0,1.5$ and $2.0 \%$ in diets. Results showed that, adding high 
level of DY, $2 \%$, recorded highest final body weight (913.26 g), growth rate $(4.72 \mathrm{~g} / \mathrm{fish} /$ day), total production $(47.5 \mathrm{~kg} / \mathrm{m} 3)$ and profit per cage $(52.21 \%)$ after 186 days. The study demonstrates that DY inclusion in the feeds of hybrid cat fish was significant. However, finding the optimal inclusion level of DY still needs more investigation of higher DY levels than 2\% (Essa et al., 2011).

Amer (2012) concluded that Diamond $V X P$ (inactive yeast a commercial product containing $100 \%$ dried Saccharomyces cerevisae distributed by DIAMOND V mils, Cidar Rapids, IOWA, and USA) could be used successfully as feed additive for feeding Nile tilapia Oreochromis niloticus without any adverse effects on their productive performance. Generally, it improves economic efficiency than control diet. Also, results obtained showed that using Diamond $V X P$ at level $(1.5 \mathrm{~g})$ had the best results on productive with levels of protein (30\%). Moreover, it could be suggested that dietary supplement with Diamond $V X P(1.5 \mathrm{~g})$ is useful in the intensive production system of Nile tilapia fish.

\section{REFERENCES}

A.O.A.C. (1990): "Association of Official Agricultural Chemists" Official methods of analysis. 15th Ed. Published by the A.O.A.C.. Benjamin Franklin Station, Washington. D.C.

Abdelhamid, A. M.; M. I. El-Barbary and M. M. M. Hasan (2012). Effect of dietary supplementation with Bio-mos ${ }^{\circledR}$ or T-protphyt 2000 with and without hormone treatment on performance, chemical composition, and hormone residues of mono-sex Nile tilapia. J. Animal and Poultry Production, Mansoura University, 3: 99-113.

Abdelhamid, A. M.; A. A. A. Soliman and N. A. Maghraby (2011). Evaluation of using the prebiotic avian plus in tilapia fish diet. Egyptian J. Nutrition and Feeding, 14 (3): 537-546.

Abdel-Tawwab, M. (2012). Interactive effects of dietary protein and live bakery yeast, Saccharomyces cerevisiae on growth performance of Nile tilapia, Oreochromis niloticus (L.) fry and their challenge against Aeromonas hydrophila infection. Aquacult Int, 20: 317-331.

Ai, Q.; Xu, H., Mai, K., Xu, W., Wang, J. and Zhang, W. (2011). Effects of dietary supplementation of Bacillus subtilis and fructooligosaccharide on growth performance, survival, non-specific immune response and disease resistance of juvenile large yellow croaker, Larimichthys crocea. Aquaculture, 317: 155-161.

Allam, H.Y.H. (2007). Physiological effects of some additives on growth, blood constituents and immunity in Nile tilapia (Oreochromis niloticus). Ph.D. Thesis, Fac. of Agric., Assiut Univ., Egypt.

Amer, A.A.A.M. (2012). Effect of different levels of protein and probiotics on productive performance of fresh water fishes. M.Sc., Fac. Agric. Kafrelsheikh Univ.

Castillo, M. (2008). Blocking pathogens with Bacillus strains. Feed-Mix, 16 (5): 19 . 
Duncan, D. (1955). Multiple range and multiple F-tests. Biometrics, 11: 1-42.

EL-Haroun, E.R. (2007). Improved growth rate and feed utilization in farmed African catfish Clarias gariepinus (Burchell 1822) thought a growth promoter Biogen ${ }^{\circledR}$ supplementation. Journal of Fisheries and Aquatic Science, 2: 319-327.

EL-Haroun, E.R., Goda, A. MA-S and Kabir Chowdhury, M.A. (2006). Effect of dietary probiotic Biogen ${ }^{\circledR}$ supplementation as a growth promoter on growth performance and feed utilization of Nile tilapia Oreochromis niloticus (L.). Aquaculture Research, 37: 1473-1480.

Essa, M.A.; Mabrouk, H.A., Mohamed, R.A. and Michael, F.R. (2011). Evaluating different additive levels of yeast, Saccharomyces cerevisiae, on the growth and production performances of a hybrid of two populations of Egyptian African catfish, Clarias gariepinus. Aquaculture, 320: 137-141.

Garling, D. L. and Wilson, R. P.(1976). Optimum dietary protein to energy ratio for Channel catfish fingerlings, Ictalurus punctatus. Journal of Nutrition., (106) : 1368-1375.

Hassan, M.E.M. (2013). Physiological Studies on Fish Production. M.Sc. Thesis, Fac. Agric., Al-Mansoura Univ.

Khalafalla, M.M.E. (2010). Growth response of Oreochromis niloticus fingerlings to diets containing different levels of Biogen. J. Agric. Res. Kafer El-Shiekh Univ., 36 (2): 97-110.

Lara-Flores, M., Olvera-Novoa, M.A., Guzmán-Méndez, B.E. and LópezMadrid, W. (2003). Use of the bacteria Streptococcus faecium and Lactobacillus acidophilus, and the yeast Saccharomyces cerevisiae as growth promoters in Nile tilapia Oreochromis niloticus. Aquaculture, 216: 193-201.

Marzouk, M.S., Moustafa, M.M. and Mohamed, N.M. (2008). The influence of some probiotics on the growth performance and intestinal microbial flora of Oreochromis niloticus. Proceedings of $8^{\text {th }}$ International Symposium on Tilapia in Aquaculture, Cairo, Egypt, pp. 1059-1071.

Mehrim, A.I. (2009). Effect of dietary supplementation of Biogen $\AA$ (Commercial probiotic) on mono-sex Nile tilapia Oreochromis niloticus under different stocking densities. J Fisher Aquat Sci., 4(6): 261-273.

Mohamed, K.A., Abdel Fattah, B. and Eid, A.M.S. (2007). Evaluation of using some feed additives on growth performance and feed utilization of monosex Nile tilapia (Oreochromis niloticus) fingerlings. Agricultural Research Journal, Suez Canal University, 7: 49-54.

New, M. B., (1987). Feed and feeding of fish and shimp. A manual on the prepartion and presenution of compound feeds for shrimp and fish in Aquaculture, United Natio Develeopment Program, FAO, Rome, PP: 14-35.

NRC (National Research Council) (1993). Nutrient requirements of fish. Committee on Animal Nutrition Board on Agriculture. National Academy Press, Washington DC., USA. 114pp.

SAS (2006). SAS/STAT Guide for personal computer. SAS Institute Inc., Cary, NC, USA. 
Waché, Y.; Auffray, F., Gatesoupe, F., Zambonino, J., Gayet, V., Labbé, L. and Quentel, C. (2006). Cross effects of the strain of dietary Saccharomyces cerevisiae and rearing conditions on the onset of intestinal microbiota and digestive enzymes in rainbow trout, Onchorhynchus mykiss, fry. Aquaculture, 258: 470-478.

Wang, Y.B., Li, J. and Lin, J. (2008). Probiotics in aquaculture: Challenges and outlook. Aquaculture, 281: 1-4.

تـأثير اختلاف مصدر ومستوى بعض الإضـافات الحيويـة العلفيـة على : 1- أداء النمـو

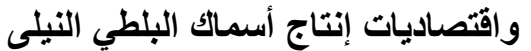

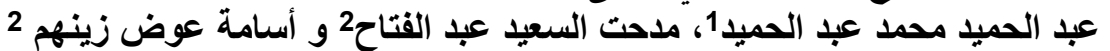

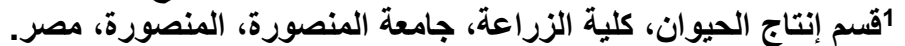

2المعمل المركزي لبحوث الثروة السمكية، مركز البحوث الزراعية، البهورة، العباسة، أبو حماد، مصر.

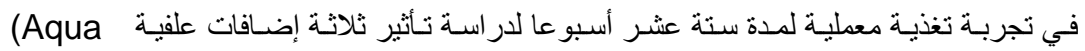

Superzyme, Garlen Allicin, and Diamond V-Original XPC)

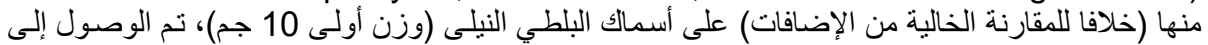

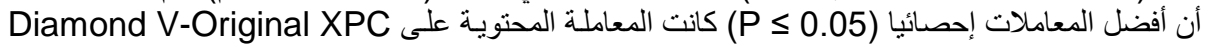

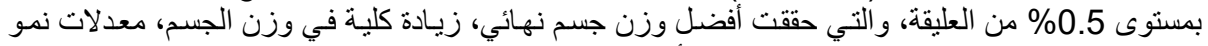

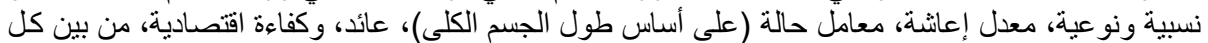

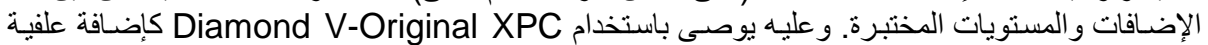
لعلائق أسماك البلطي النيلى بتركيز 0.5\% من العليقة لتحسين أداء النمو والحياتية واقتصاديات الإنتاج.

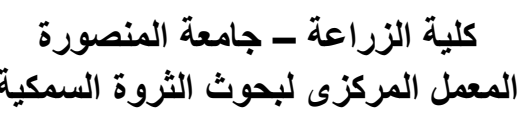

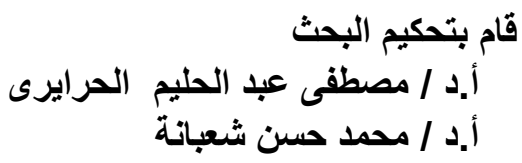

